\title{
Role of Diffusion Weighted MRI in Maple Syrup Urine Disease: Rare Cause of Neonatal Metabolic Encephalopathy
}

\author{
Shah $D^{1}$, Nirula $A^{2}$, Shah $D^{3}$ \\ ${ }^{1}$ Dr. Diva Shah, MBBS, MD, Assistant Professor, ${ }^{2}$ Dr. \\ Apoorv Nirula, MBBS, MD Resident, ${ }^{3}$ Dr. Dwiti Shah, \\ MBBS, MD Resident. All from the Department of \\ Radiodiagnosis, Pramukhswami Medical College, \\ Shrikrishna Hospital, Gokal Nagar, Karamsad, Anand, \\ Gujrat, India.
}

\section{Introduction}

$\mathrm{M}$ aple syrup urine disease is an autosomal recessive disease. The purpose of this case report is to educate with typical MRI picture in case of metabolic encephalopathy for diagnosis and treatment of MSUD in early neonatal period. The commonest and severest form of the disease is the classic type, which is characterized by a neonatal onset of encephalopathy. Acute phase of classical MSUD shows a typical distribution of cytotoxic oedema on diffusion-weighted imaging with remarkably low corresponding ADC values, known as "MSUD oedema". Early diagnosis may alter the course of the disease and improves child's condition.

\section{The Case}

A full term male infant child with seven days life, having birth weight of $3.75 \mathrm{~kg}$ and uneventful birth history with apgar score of 9/10. He presented with feeding difficulties, weak cry after two days of birth and followed by multiple episodes of generalized tonic clonic seizures, apathy and apnea. Patient was kept on ventilator under sedation. On physical examination, there was no cyanosis, no icterus or pallor. His mother has bad obstetric history with multiple fetal loss and loss of siblings (G7 P2 A3 L2). Clinically, it was thought of metabolic encephalopathy v/s severe sepsis. Patient was kept on ventilator under sedation.

CSF examination was done and it revealed normal total WBC count and differential count with raised ketone. Biochemical examinations showed hypoglycemia and metabolic ketoacidosis.

\author{
Address for correspondence \\ Dr. Diva Shah \\ E-mail:drdiva1923@yahoo.co.in
}

This work is licensed under a Creative Commons Attribution 3.0 License.

\begin{abstract}
The newborn presenting with neurological symptoms such as seizures or lethargy due to inborn error of metabolism is an important problem. Maple syrup urine disease (MSUD) is an inherited genetic disease, caused by a deficiency of the catalytic components of a-ketoaciddehydrogenase complex, which is responsible for the catabolism of branched-chain amino acids. The purpose of this case report is to show diffusion-weighted imaging (DWI) MRI findings of acute phase of classic form of MSUD in a newborn although this imaging findings are rare but very typical, known as "MSUD oedema".
\end{abstract}

Key words: Maple syrup urine disease, MSUD oedema, Classic form, metabolic encephalopathy

Brain MRI done at 10 days of life, showed symmetrical hyper signal lesions on DWI and corresponding hypo signal on ADC maps in bilateral thalami, posterior limb of internal capsule, bilateral cerebral peduncles (Figure 2), pons and medulla and also involving deep cerebellar white matter (Figure 1) as well as perirolandic white matter. Faint hypersignal intensity seen on T2W images involving ponto-medullary junction and in bilateral thalami and these areas show faint hypo signal intensity on T1W images (Figure 3).

Chromatographic analysis showed an increase of the branched-chain amino acids in the plasma with markedly elevated levels of leucine $=2520 \mu \mathrm{mol} / \mathrm{L}$ (normal values: $<385 \mu \mathrm{mol} / \mathrm{L}$ ); isoleucine $=648 \mu \mathrm{mol} / \mathrm{L}$ (normal values: $26-91 \mu \mathrm{mol} / \mathrm{L}$ ) and valine $=645 \mu \mathrm{mol} / \mathrm{L}$ (normal values $<306 \mu \mathrm{mol} / \mathrm{L}$ ).

Leucine/ Alanine ratio was 24.63 (normal range is $0.12-0.53$ ), and Leucin/Tyrosin ratio was 77.10 (normal range is $0.50-3.50$ ). This pattern is suggestive of MSUD. Repeat chromatography was not possible as clinical condition of the baby became worsened and he died at $11^{\text {th }}$ day of life.

\section{How to cite this article ?}

Shah D, Nirula A, Shah D. Role of Diffusion Weighted MRI in Maple Syrup Urine Disease: Rare Cause of Neonatal Metabolic Encephalopathy. J Nepal Paediatr Soc 2013;33(3):230-233. 


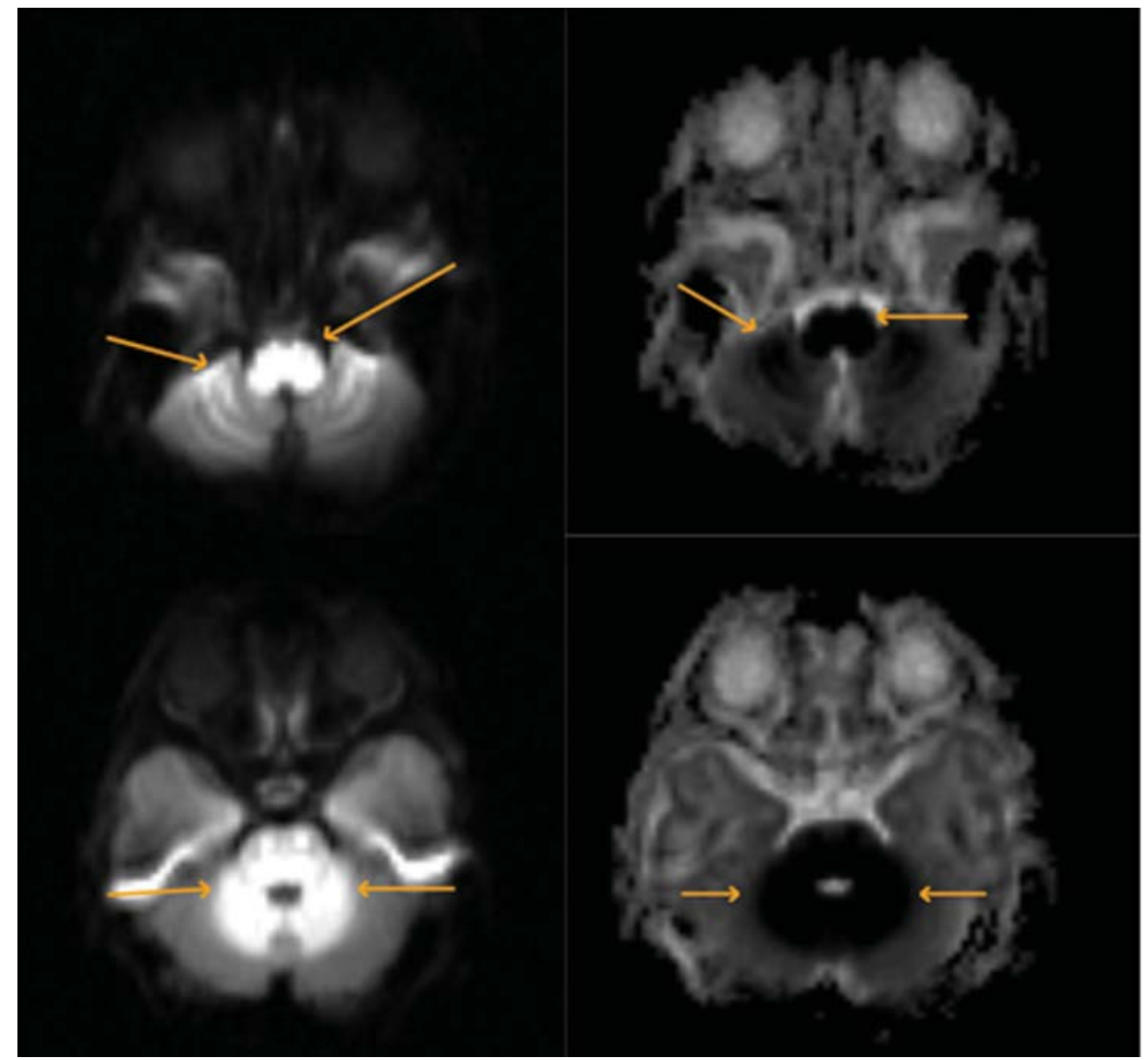

Fig 1: DW and ADC images showing marked water motion restriction in Medulla, Dorsal Pons and bilateral deep cerebellar white matter.
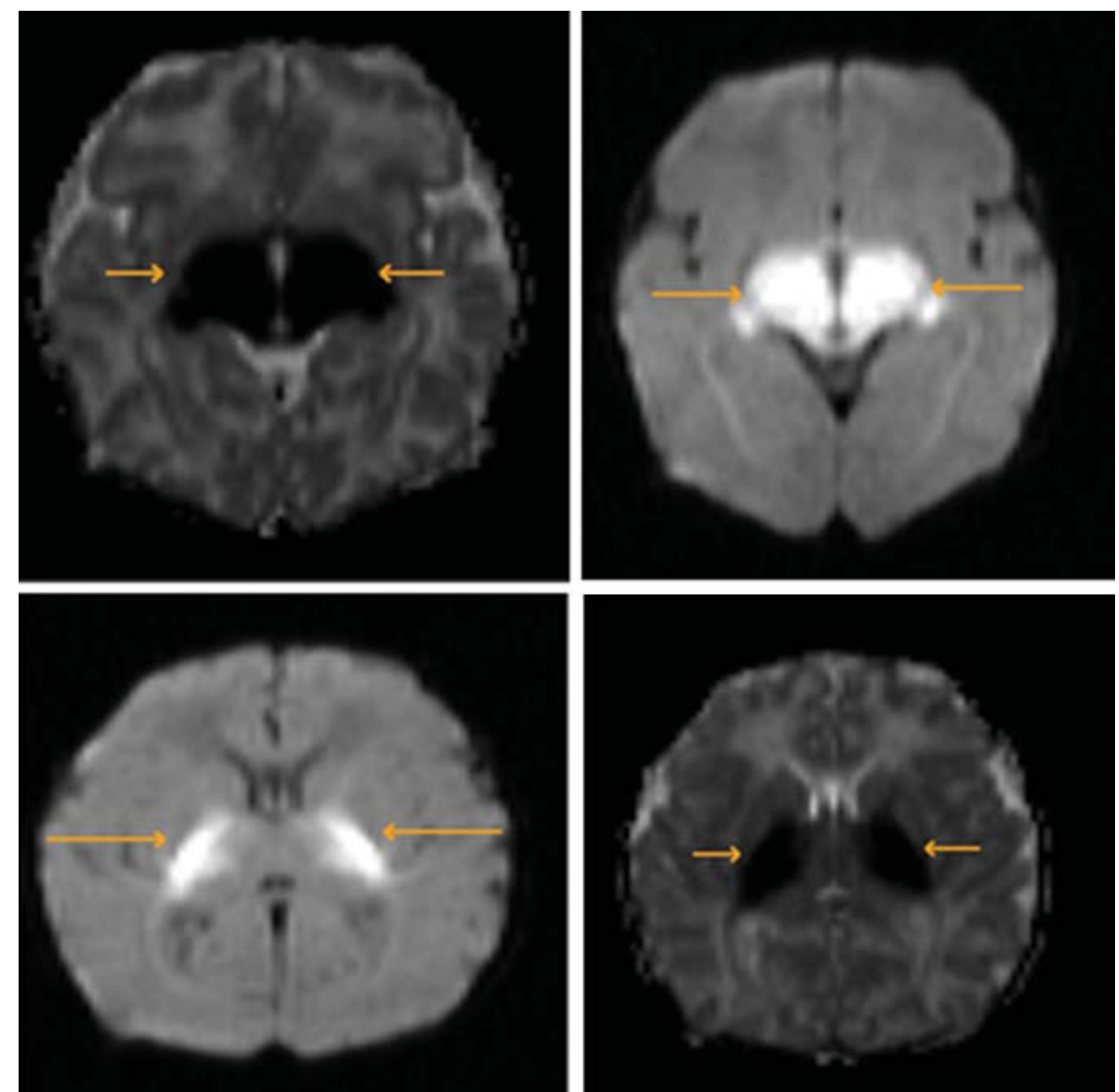

Fig 2: DW and ADC images showed marked water motion restriction in Bilateral Thalamus and Posterior Limb of Internal Capsule 

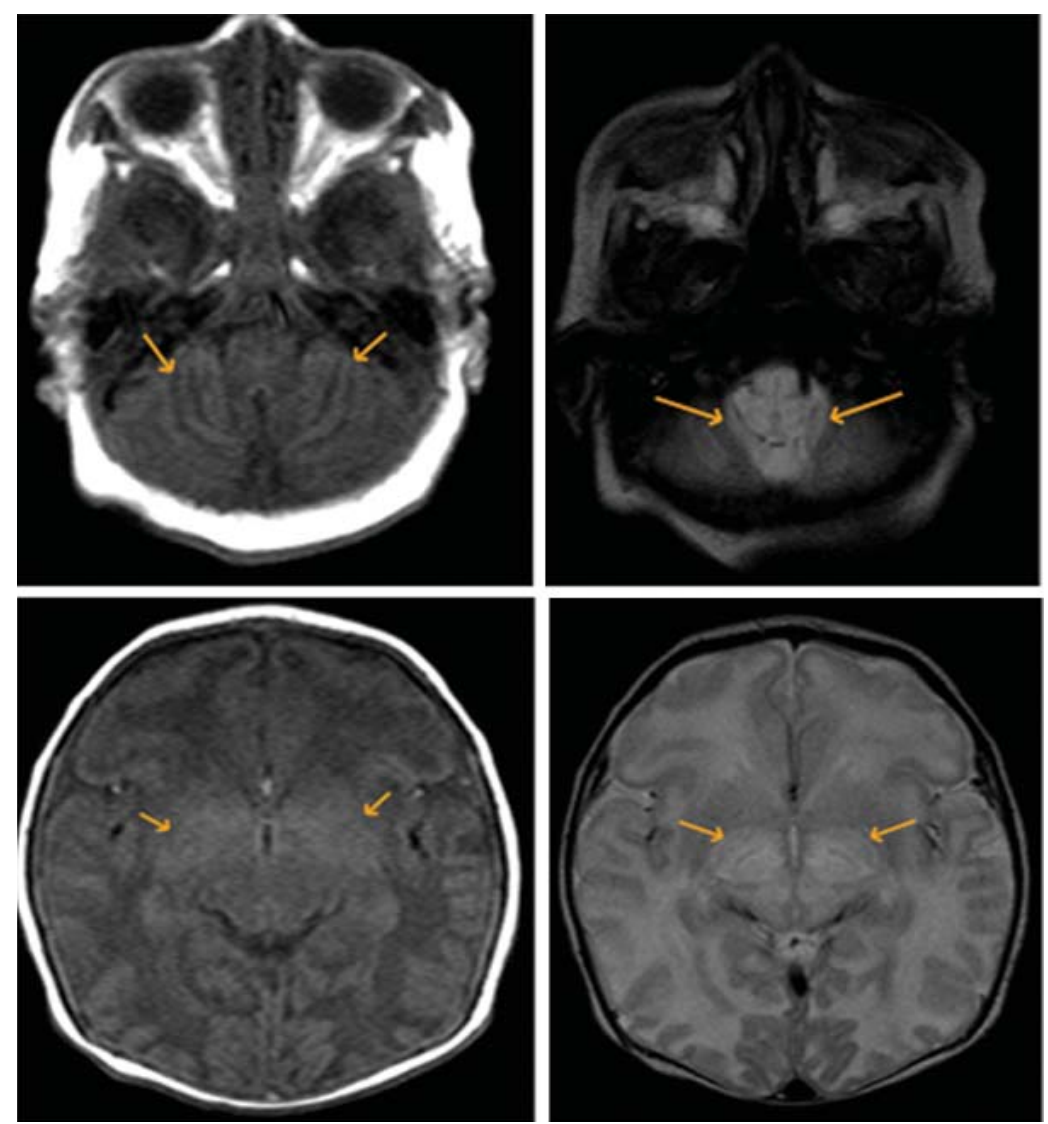

Fig 3: T1W and T2W images showing altered signal intensity in Bilateral Cerebellar Folia and Bilateral Thalamus

\section{Discussion}

Maple syrup urine disease is an autosomal recessive disorder caused by a block in breakdown of the three-branched chain amino acids (BCAAs), leucine, isoleucine, and valine due to genetic defect of mitochondrial branched chain alpha ketoaciddehydrogenese complex. This enzyme deficiency leads to the accumulation of toxic levels of branched chain amino acids and branched chain alpha ketoacidosis in the body resulting in severe metabolic acidosis, neurological deterioration and the characteristic maple syrup urine odor ${ }^{1}$. The condition has incidence of approximately 1 out of $1,20,000-5,00,000$ infants worldwide ${ }^{2}$

Each parent of a child with MSUD carries a defective recessive gene for MSUD, along with a normal gene $^{3}$. The parents of a child with MSUD have a 1 in 4 chance during each pregnancy that the baby will receive a defective gene from each parent and have MSUD; a 2 in 4 chance the baby will receive one defective and one normal gene becoming a carrier of MSUD; a 1 in 4 chance that the baby will receive two normal genes"3. In our case patient's mother had a bad obstetric history with multiple foetal loss and loss of siblings; she has only two live children out of seven.
MSUD is expressed in different forms as classic, intermittent, intermediate, thiamine responsive and dihydrolipoyldehydrogenase (E3)-deficient ${ }^{1}$. The classic form presents in the first week of life with poor feeding, vomiting and leading to convulsions, lethargy and coma, so was in our case also. Clinically, there is hypertonicity and muscular rigidity with opisthotonus posture. Neurological findings are often mistaken for sepsis or meningitis, and death occurs if untreated ${ }^{4}$. In our case of also we thought of clinically sepsis only.

Conventional MRI during acute metabolic decompensation phase shows characteristic abnormal hypersignal intensity on $\mathrm{T} 2 \mathrm{~W}$ images, involving the cerebellar white matter, the posterior brainstem, the cerebral peduncles, the thalami, the posterior limbs of the internal capsules and perironaldic region and DW images show acute restriction in these areas. Analysis of $A D C$ in these regions revealed a markedly low value (reduced by approximately $80 \%$ ). This indicates that MSUD oedema in the first postnatal days is an intracellular oedema (so-called cytotoxic oedema) ${ }^{2,5,6,7}$. In the present case we had classical distribution of cytotoxic edema.

DWI proves to be more sensitive to the MSUD- 
related brain alterations in the acute stage than conventional MRI in showing hyperintensity of the MSUD oedema ${ }^{7,8,9}$, Myelinated white matter changes during the acute phase of MSUD are characterized by a decreased value of ADC that may be a result of neurotransmitter disorders, such as an increase in glutamate, an impaired energy metabolism associated with increases in brain lactate and decreased synthesis of lipid and proteolipid proteins ${ }^{2,6,10}$.

Follow-up DWI associated with amino acid plasma level measurements may be of predictive value for the clinical outcome and the efficacy of treatment ${ }^{5}$. In our case child was diagnosed having typical MSUD cytotoxic edema on $10^{\text {th }}$ day of birth, but follow up MRI study was not possible as patient died on $11^{\text {th }}$ day of birth.

The unfavorable outcome in our case report may be justified because the diagnosis and specific treatment were delayed; with remarkably altered amino acid plasma levels.

In conclusion, acute phase of classical MSUD shows a typical distribution of cytotoxic edema on diffusionweighted imaging with remarkably low corresponding ADC values, known as MSUD edema. This MR picture should kept in mind for earliest diagnosis and treatment of MSUD in first week of birth of neonate. Early diagnosis and management of neonatal metabolic encephalopathy may prevent some or all of this morbidity, and also permits the parents to be informed about the chances of having other affected children.

Acknowledgement: Special thanks to Dr. C Raychaudhari, Head, Department of Radiodiagnosis, Pramukhswami Medical College for constant support and inspiration.

\section{References}

1. Wajanat Jan, Robert A. Zimmerman, Zhiyue J. Wang, Gerard T. Berry, Paige B. Kaplan, Edward M. Kaye MR diffusion imaging and MR spectroscopy of maple syrup urine disease during acute metabolic decompensation. Neuroradiology 2003;45:393-9.

2. José Roberto Lopes Ferraz-Filho, Valdeci Hélio Floriano, Marcelo Bianco Quirici, Regina Pires de Albuquerque, Antônio Soares Souza, Contribution of the diffusion-weighted MRI in the diagnosis and follow-up of encephalopathy caused by maple syrup urine disease in a full-term newborn. Arq Neuropsiquiatr 2009;67:719-723.

3. Weber, Robert C. Cornelia de Lange Syndrome / Maple Syrup Urine Disease / Rubinstein-Taybi Syndrome: etiologies and indicators: 2005 Part 1 The Free Library (September, 22).
4. Simon E, Flaschker N, Schadewaldt P, Langenbeck $U$, Wendel U. Variant maple syrup urine disease (MSUD)_The entire spectrum. J Inherit Metab Dis 2006;29(6):716-24.

5. Jong Su Ha,Taik-Kun Kim,Baik-Lin Eun,Hee Sun Lee,Ki Yeol Lee,Hae Young Seol, Sang Hoon Cha, Maple syrup urine encephalopathy: a follow-up study in the acute stage using diffusion-weighted MRI. Pediatr Radiol 2004;34:163-166.

6. Felber SR, Sperl W, Chemelli A, Murr C, Wendel U. Maple syrup urine disease: metabolic decompensation monitored by proton magnetic resonance imaging and spectroscopy. Ann Neurol 1993;33:396-01.

7. Righini A, Ramenghi LA, Parini $R$, et al,Water apparent diffusion coefficient and $T 2$ changes in the acute stage of maple syrup urine disease: evidence of intramyelinic and vasogenic-interstitial edema. $J$ Neuroimaging 2003;13:162-165.

8. Kevin A. Strauss, Donna L. Robinson, Erik G. Puffenberger, D. Holmes Morton. Diagnosis and Treatment of Maple Syrup Disease: A Study of 36 Patients. Pediatrics 2002; 109: 999 -1008

9. Philippe Jouvet, Pierre Rustin, Deanna L. Taylor, Jennifer M. Pocock, Ursula Felderhoff-Mueser, Nicholas D. Mazarakis. Branched chain amino acids induce apoptosis in neural cells without mitochondrial membrane depolarization or cytochrome $C$ release: implications for neurological impairment associated with maple syrup urine disease. Mol Biol Cell 2000;11:1919-1932.

10. Cavalleri F, Beradi A, Burlina AB, Ferrari F, Mavilla L, Diffusion-weighted MRI of maple syrup urine disease encephalopathy. Neuroradiology 2002;44:499-502.

\author{
Abbreviations used: \\ MRI-Magnetic Resonance Imaging \\ DWI-Diffusion Weighted Imaging \\ ADC-Apparent Diffusion Coefficient map \\ MSUD-Maple Syrup Urine Disease \\ G- Gravida; P- para, A- abortion and L - Live children \\ CSF- Cerebrospinal Fluid \\ WBC: White Blood Cell count
}

BCAA: Branched Chain Amino Acids 